\title{
Effect of linear density of states on the quasi-particle dynamics and small electron-phonon coupling in graphite
}

\author{
C. S. Leem ${ }^{1}$, B. J. Kim², Chul Kim¹, S. R. Park ${ }^{1}$, T. Ohta ${ }^{3}$, A. Bostwick ${ }^{3}$, \\ E. Rotenberg ${ }^{3}$, H. -D. Kim ${ }^{4}$, M. K. Kim ${ }^{1}$, H. J. Choi ${ }^{1}$, and C. Kim ${ }^{1, *}$ \\ ${ }^{1}$ Institute of Physics and Applied Physics, Yonsei University, Seoul, Korea \\ ${ }^{2}$ School of Physics and Center for Strongly Correlated Materials Research, Seoul National University, Seoul, Korea \\ ${ }^{3}$ Advanced Light Source, Lawrence Berkeley National Laboratory, Berkeley, California 94720, USA and \\ ${ }^{4}$ Pohang Accelerator Laboratory, Pohang 790-784, Korea
}

(Dated: November 1, 2018)

\begin{abstract}
We obtained the spectral function of very high quality natural graphite single crystals using angle resolved photoelectron spectroscopy (ARPES). A clear separation of non-bonding and bonding bands and asymmetric lineshape are observed. The asymmetric lineshapes are well accounted for by the finite photoelectron escape depth and the band structure. The extracted width of the spectral function (inverse of the photohole life time) near the $K$ point is, beyond the maximum phonon energy, approximately proportional to the energy as expected from the linear density of states near the Fermi energy. The upper bound for the electron-phonon coupling constant is about 0.2, a much smaller value than the previously reported one.
\end{abstract}

PACS numbers: 74.25.Jb, 63.20.Ls, 79.60.-i

Recent discoveries of novel physical properties in carbon-based materials such as superconductivity $\underline{1,2,3,4,5}$ and massless Dirac Fermions $\underline{6}$ brought renewed interest in the electronic structure of graphite $\frac{7.8}{}$. The peculiarity of the electronic structure of graphite has two aspects: graphite is extremely two dimensional and is a semi-metal. These facts make it fundamentally interesting to study how dimensionality affects the dynamics of the doped carriers and how the carriers in graphite intercalated compounds (GICs) couple to the mediating bosons. In fact, there is a long standing issue in regards to the carrier dynamics in graphite, that is, whether the carriers are Fermi-liquid-like or not. This question motivates experimental studies of electronic structures of these materials by using, for example, angle-resolved photoemission spectroscopy (ARPES) and one can find a long history in the ARPES studies on graphite single crystals $7,8,9,10,11,12,13,14,15$. In addition, studies of graphite-related materials such as single $\frac{16}{\underline{ }}$ and bilayer graphene $\frac{17}{}$ and GICs $\frac{18,19}{19}$ can be found.

High quality ARPES data from graphite is difficult to obtain despite graphite's two dimensional, inert nature. The problems associated with ARPES experiments on graphite are due mostly to difficulty in proper surface preparation and to some extent to the low quality of the single crystals. For example, the extreme twodimensional nature of graphite inevitably produces small flakes (many are small enough to be seen only under microscopes) on the cleaved surfaces which ruins the momentum resolution in ARPES. Such difficulties prevented one from obtaining good quality data to extract reliable information on the many-body interactions such as electron-phonon coupling (EPC). Therefore, unless such difficulties are overcome, reliable information on manybody interactions can not be extracted from the data. As a result, the experimental data in regards to the electron lifetime have been obtained mostly by time-resolved photoelectron spectroscopy on highly oriented pyrolytic graphite 20,21 .

Motivated by the renewed interest in the carrier dynamics in graphite, we have performed ARPES studies on graphite single crystals. Our goal was to extract reliable quantitative information on the EPC from ARPES data. To overcome the above mentioned difficulties, we exploited micro-spot ARPES and high quality natural graphite single crystals, and were able to obtain ARPES data with high enough quality to reliably extract quantitative information on the electron-phonon coupling. In addition, we successfully applied the finite photoelectron escape depth and band structure effects in graphite to the analysis of the ARPES spectral functions for the first time (to our knowledge). This allowed us to obtain an accurate measure of the EPC in graphite by using ARPES.

ARPES experiments were performed at the beam line $3 \mathrm{~A} 1$ of the Pohang Accelerator Laboratory (preliminary) and 7.0.1 of the Advanced Light Source (main results). High quality natural graphite single crystals with sizes larger than $1 \mathrm{~cm}$ were cleaved ex situ. The samples were subsequently introduced to the vacuum chamber and annealed for half an hour at about $900^{\circ} \mathrm{C}$ in a vacuum better than $6.0 \times 10^{-10}$ Torr. ARPES measurements were performed at $25 \mathrm{~K}$ with an energy resolution of about 40 meV which mostly stemmed from $0.0075 \AA^{-1}$ momentum resolution in combination with the fast dispersion of the bands. The pressure was better than $5.0 \times 10^{-11}$ Torr. For the experiment, we exploited the small beam spot $(\approx 40 \mu \mathrm{m})$ to locate a flat region without flakes. A photon energy of $85 \mathrm{eV}$ was used so that $k_{z}$ was near $K$. Electronic structure calculation was done by using the SIESTA $\operatorname{code}^{22}$ based on pseudo-potential method.

Fig. 1(a) shows the calculated electronic band dispersion of graphite along the high symmetry lines, $\Gamma-\mathrm{M}-\mathrm{K}-\Gamma$ (solid lines) and A-L-H-A (dashed). Also shown in the figure as an intensity map is the experimental data with 

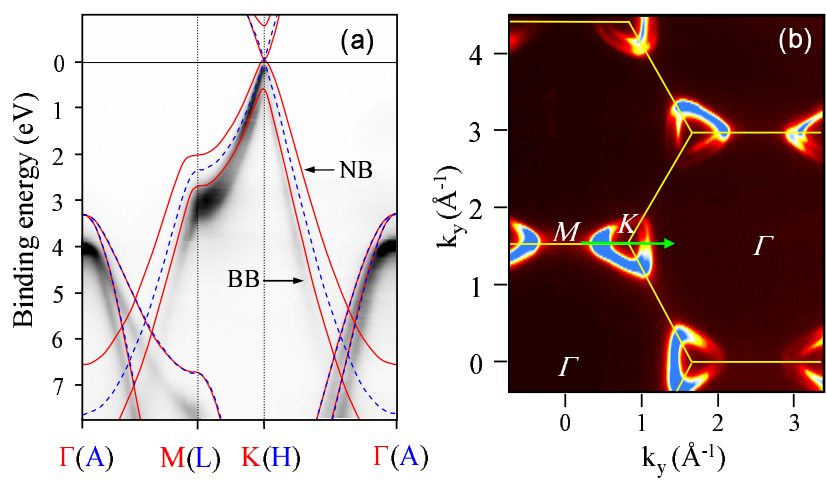

FIG. 1: (a) Comparison of experimental data and calculated band structure of graphite along the high symmetry lines. (b) Intensity map at a constant binding energy of $1.75 \mathrm{eV}$. Brillouin zones are drawn as solid lines.

the photon energy tuned for $k_{z}=\mathrm{K}$. Other than the fact that the calculated band structure has to be expanded, which is well known ${ }^{7}$, theoretical and experimental results match well. The two bands near the Fermi energy are $p_{z}$-derived $\pi$ bands and are split due to the inter-layer interaction, resulting in anti-bonding $(\mathrm{AB})$, non-bonding (NB) and bonding (BB) bands. Among them, NB and $\mathrm{BB}$ are occupied and can be seen by ARPES. The splitting $\omega_{B}$ between the $\mathrm{NB}$ and $\mathrm{BB}$ is about $0.7 \pm 0.1 \mathrm{eV}^{23}$. The data can also be plotted in momentum space at a constant binding energy (1.75 eV) as shown in Fig. 1(b). The plot shows an apparent hexagonal Boulliouin zone expected for graphite. It is also clearly seen that rounded triangles around the $\mathrm{K}$ points are split. The bands have approximate linear dispersion with point-like Fermi surfaces at the $\mathrm{K}$ points, resulting in an approximate linear electronic density of states near the Fermi energy (linear DOS).

Fig. 2(a) shows high resolution data taken along the M-K- $\Gamma$ line as marked in Fig. 1(b). The two peaks are sharp and clearly split. We note that there are no defectinduced states as reported in Ref ${ }^{24}$. In addition, the background at high binding energies is very small, in strong contrast to the previously reported data $89,10,11,15$. We attribute these to the high quality of the crystals used in our experiment. Fig. 2(b) shows the energy distribution curve (EDC) at the K-point (arrow in panel (a)). One peculiar aspect of the data is that the region between the two peaks are somewhat filled up. As a result, the BB has a tail on the lower binding energy side contrary to the usual case. This aspect of the data can be understood as follows. Even though we tuned the photon energy to the $\mathrm{K}$ point, the finite escape depth of the photoelectron introduces an uncertainty in $k_{z}, \Delta k_{z}=1 / \mu$ where $\mu$ is the escape depth. This uncertainty in $k_{z}$ depicted in Fig. 2(c) necessarily brings in broadening due to the $k_{z}$ dispersion, which is known as the photoelectron life time. Note that the NB (BB) has its dispersion maximum (minimum) at $k_{z}=0$ and as a consequence the broadening effect will be one-sided towards the band
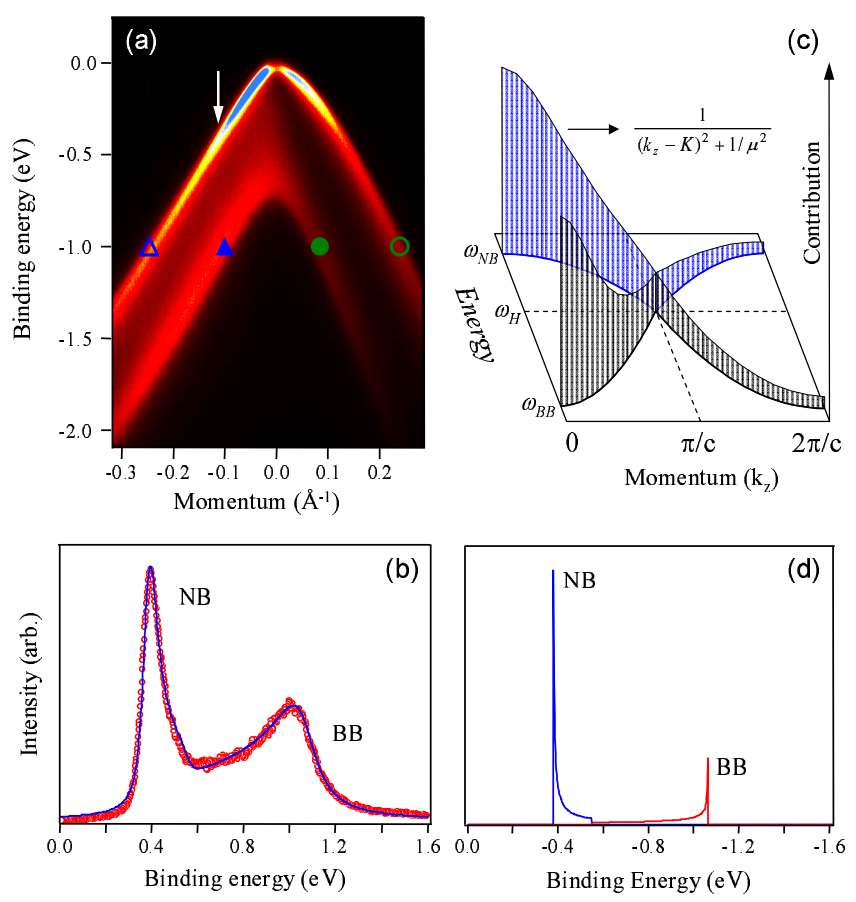

FIG. 2: (a) High resolution ARPES data taken along the M$\mathrm{K}-\Gamma$ as marked in Fig. 1(b). (b) A typical EDC (circles), taken at the $k$ value marked in panel (a) and the fit (line) using the model discussed in the text. (c) Schematic of the dispersions along the $k_{z}$ direction for non-bonding (upper curve) and bonding (lower curve) bands. Due to the finite escape depth $(\mu)$, a range of $k_{z}$ contributes to the spectral functions. The contribution probability of each $k_{z}$ is represented by the height of the graph. (d) Resulting spectral function near $\mathrm{K}$ point without lifetime broadening.

position at $k_{z}=\pi / c$ where the two bands collapse. Fig. 2 (d) depicts a model spectral function when all these effects are accounted for. Only when such effects are considered can one reliably extract the lifetime broadening. We used $\mu=7 \AA$ for the fitting 25 and the model function in Fig. 2(d) is convolved with a Voigt function with the Gaussian width set to the total energy resolution of 40 meV. A typical fit is shown in Fig. 2(b) as the solid line.

Before we look into the low energy dynamics of graphite, it is necessary to discuss how the couplings to phonons and other low energy excitations are affected by the linear DOS of graphite. The EPC in metallic systems is well understood 26 and has been extensively studied recently by using ARPES 27,28. However, there is a relative lack of understanding of EPCs in semi-metals. In fact, to our best knowledge, EPC effects on the ARPES lineshape for semi-metals with linear DOS have not been discussed. As a consequence, models derived for metals have been improperly used in graphite studies $\stackrel{8}{\text {. }}$.

To understand the EPC in a system with a linear DOS, we evaluate the Feynman diagram for a single phonon process shown in Fig. 3(a). We first look at a single band system for simplicity and will extend the analysis to the graphite case. The contribution from the process 
to the imaginary part of the self energy $\Sigma_{\text {ep }}^{\prime \prime}$ due to EPC at $T=0$ is $\underline{\underline{29}}$

$\Sigma_{\text {ep }}^{\prime \prime}\left(\omega_{k}\right)=\sum_{\nu} \int\left|<k^{\prime}, q\right| H_{1}|k, 0>|^{2} f\left(-\omega_{k^{\prime}}\right) \delta\left(\omega_{k}-\omega_{k^{\prime}}-\omega_{\nu, q}\right) d k$

where $k$ and $k^{\prime}$ are crystal momenta of the holes and $f$ Fermi-Dirac function at $T=0$, respectively. The phonon momentum is $q=k-k^{\prime}$, and $\nu$ represents the phonon mode. $H_{1}$ is the Hamiltonain for the EPC, and it can be written as

$$
H_{1}=g c_{k-q}^{\dagger} c_{k} b_{q}^{\dagger}
$$

where $g$ is the transition probability amplitude for particular $k$ and $q$. Assuming Einstein phonons and constant $g$,

$$
\begin{array}{r}
\int\left|<k^{\prime}, q\right| g c_{k-q}^{\dagger} c_{k} b_{q}^{\dagger}|k, 0>|^{2} \delta\left(\omega_{k}-\omega_{k^{\prime}}-\omega_{\nu, q}\right) d k^{\prime} \\
=g^{2} \int \delta\left(\omega_{k}-\omega_{k^{\prime}}-\omega_{\nu, q}\right) d k^{\prime}=g^{2} \mathcal{D}\left(\omega_{k}-\omega_{\nu}\right)
\end{array}
$$

where $\mathcal{D}$ is the electronic DOS. The expression for $\Sigma_{\mathrm{ep}}^{\prime \prime}$ then becomes

$$
\Sigma_{\text {ep }}^{\prime \prime}(\omega)=\sum_{\nu} g^{2} \mathcal{D}\left(\omega-\omega_{\nu}\right) f\left(\omega_{\nu}-\omega\right)
$$

For a metal, $\mathcal{D}$ is approximately constant in the immediate vicinity of $E_{F}$ and above equation reduces to the expected step function. On the other hand, for a linear DOS, $\Sigma_{\text {ep }}^{\prime \prime}(\omega)$ increases linearly beyond the phonon energy as shown in Fig. 3(b) ${ }^{30}$.

We may now extend the analysis to the two band case. The EPC Hamiltonian becomes

$$
H_{1}=\sum_{j} g_{i j} c_{k-q, j}^{\dagger} c_{k, i} b_{q}^{\dagger}
$$

where $i$ and $j$ are band indices with $i$ representing the initial band. $g_{i j}$ with $i=j$ is the EPC for intra-band transition while $g_{i j}$ with $i \neq j$ for inter-band transition. The result is shown schematically in Fig. 3(c) $\underline{30}$. Note that $\Sigma_{\text {ep }}^{\prime \prime}$ for the NB has an upward kink at the energy where the BB starts. The kink and slopes of the self energy curves carry the information on the EPC matrix element $g_{i j}$.

The difficulty in extracting the EPC constant $\lambda$ comes from the fact that it is almost impossible to guess the bare bands. In metals, the bare band can be approximated by a linear band (possibly with an additional small parabolic term) over the energy window of interest which can be obtained by fitting the experimental dispersion. This method, however, cannot be used for semi-metals and insulators for an obvious reason. In fact, the NB band of graphite near $\mathrm{K}$ does not form a Dirac cone but has a fairly strong curvature as is well known from the band calculation. Therefore, the standard method of obtaining the real part of the self energy $\Sigma^{\prime}$ by taking (a)

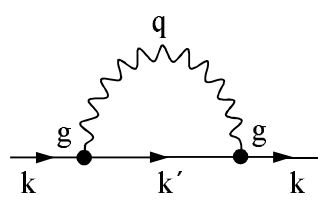

(b)

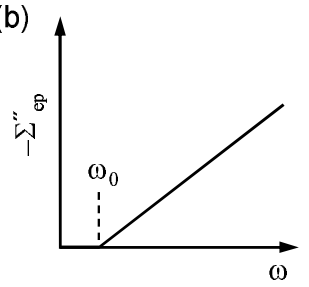

(c)

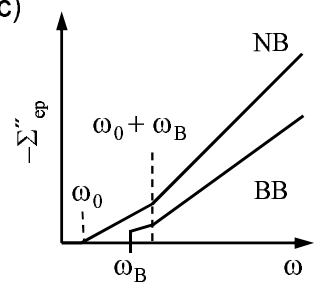

(d)

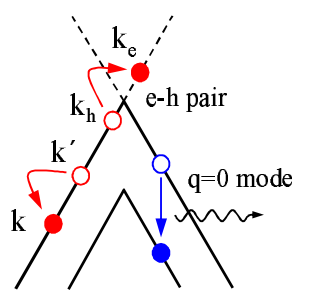

FIG. 3: (a) Feynman diagram for the EPC under consideration. (b) Schematic of the $\Sigma^{\prime \prime}$ for the phonon decay for a single band system with a linear DOS. (c) $\Sigma^{\prime \prime}$ for a double band system. (d) Other possible decay channels for photoholes, red for decay through electron-hole creation and blue for $q=0$ mode decay. Note that $k^{\prime}$ and $k_{h}$ can be changed for the electron-hole creation case.

the difference between the bare band and the experimental dispersion can not be used. Alternatively, $\Sigma^{\prime}$ can be obtained from $\Sigma^{\prime \prime}$ through the Kramers-Kronig relation. Then the EPC constant $\lambda$ can be extracted from the real part of the self energy using the following formula ${ }^{26}$.

$$
\lambda=-\left.\frac{\partial \Sigma_{\mathrm{ep}}^{\prime}(\omega)}{\partial \omega}\right|_{\omega=0}
$$

We point out that this method usually requires higher quality of data compared to the standard method to extract the similar quality of information.

Finally, we discuss other decay channels (see Fig. 3(d)). The photo-hole may decay by creating an electron-hole pair or by exciting a $q=0$ mode in addition to emitting a phonon. For the decay through electron-hole pair creation, the energy and momentum conservations mandate $\Delta \omega=\omega_{k^{\prime}}-\omega_{k}=\omega_{e}-\omega_{h}$ and $\Delta \mathbf{k}=\mathbf{k}^{\prime}-\mathbf{k}=\mathbf{k}_{e}-\mathbf{k}_{h}$. Note that both $\mathbf{k}^{\prime}$ and $\mathbf{k}$ are in the same (lower) Dirac cone while $\mathbf{k}_{e}$ and $\mathbf{k}_{h}$ are in different Dirac cones. In such case, for the equations to be met, both $\mathbf{k}^{\prime}-\mathbf{k}$ and $\mathbf{k}_{e}-\mathbf{k}_{h}$ vectors should lie in the steepest decent line on the 2 dimensional conical dispersion (the configuration illustrated in the figure) and the available phase space volume for the transition in $\Delta \omega-\Delta \mathbf{k}$ space is zero $\underline{30}$. For the $q=0$ mode decay, the contribution is considered to be photo-hole momentumindependent as the two bands are almost parallel to each other. Note that NB band is not susceptible to $q=0$ mode decay as one can see in the figure, giving a constant upward shift only to $\Sigma^{\prime \prime}$ of the BB.

With the finite escape depth considered, the half width of the Lorentzian is extracted and plotted in Fig. 4(a). Filled and empty symbols represent the BB and NB, respectively as marked in Fig. 2(a). Overall, the 

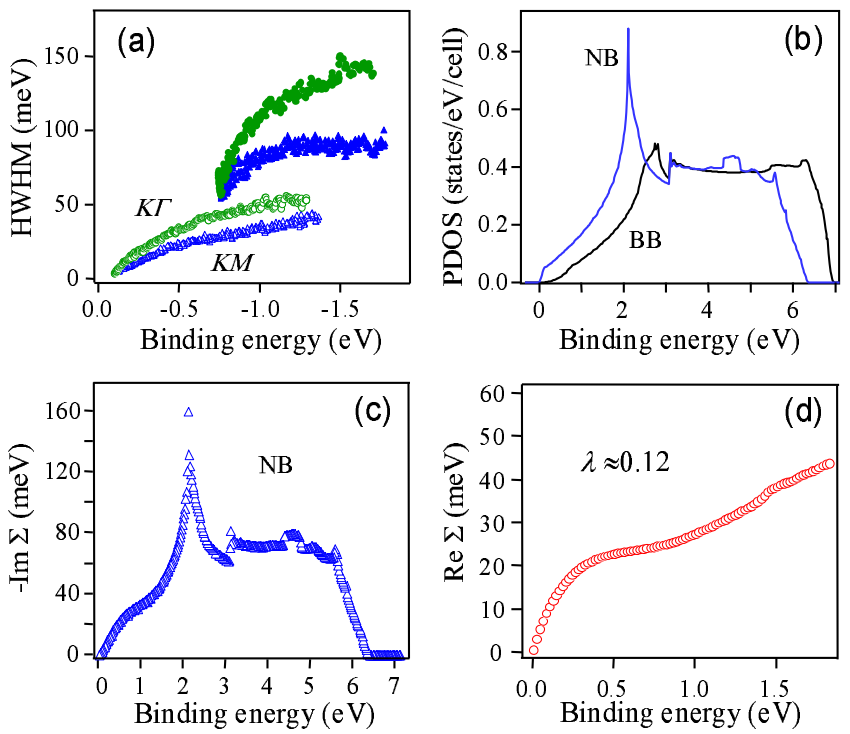

FIG. 4: (a) $\Sigma^{\prime \prime}$ (half of the Lorentzian width) vs. binding energy. (b) Calculated PDOS of the NB and BB. (c) $\Sigma^{\prime \prime}$ for the NB over the entire energy range range by combining the experimental data and the calculated PDOS. (d) $\Sigma^{\prime}$ obtained by Hilbert transforming the $\Sigma^{\prime \prime}$ in panel (c).

width monotonically increases without particular features. There are smooth slope changes at around 0.4 $\mathrm{eV}$ which roughly coincide with energy where $k_{z}$ dispersion of the NB saturates. Therefore, we attribute this to the yet unaccounted band structure (in combination with the finite escape depth) effect ${ }^{31}$. When such an effect is considered through additional modeling, we expect the width will increase more linearly. While the values for the NB, when converted to life time, are reasonably compatible with the values obtained by time resolved techniques ${ }^{20,21}$, they are much smaller than the previously reported values 8 .

From the measured widths, we find the the following additional facts. Firstly, the fact that the observed widths do not show a high order behavior $\left(\sim \omega^{2}\right)$ reveals that the EPC is the dominant decay channel for the photo-holes. This is expected because the available phase space is very small as discussed above. Secondly, no (or very weak at most) kinky feature at the optical phonon energy $(\sim 0.2 \mathrm{eV})$ is observed. It was previously reported that a strong EPC was observed in the imaginary $\operatorname{part}^{8}$ of the self energy. Our observation shows it is not the case (at least near the K point). Thirdly, the inter-band decay, if exists, is much weaker than the intra-band evidenced by the lack of an upward kink in the NB band curve at the onset energy of BB (see Fig. $3(\mathrm{c}))$. This allows us to have a convenience of treating the NB and BB independently. As will be clear, this makes the task of calculating the EPC constant much simpler. Fourthly, as the inter-band decay is very small, the width of the $\mathrm{BB}$ at the onset energy can not be explained by the EPC. It has to come from another mechanism such as the $q=0$ mode decay as discussed earlier which is not well-understood. Lastly, the slopes of the curves are somewhat $k$-dependent (that is, the slopes for the $\mathrm{K}-\mathrm{M}$ cut are steeper). This $k$-dependence suggests that there is $k$-dependent EPC in the system. Such behavior is indeed predicted theoretically ${ }^{32}$ and is also observed in the study of a single layer graphene ${ }^{33}$.

Even though the constant offset in the $\Sigma^{\prime \prime}$ of the BB is not well-understood, one can estimate the EPC of the NB as the inter-band transition is weak. As discussed above, calculating the EPC constant $\lambda$ requires knowledge of $\Sigma^{\prime \prime}$ over the entire energy range. As it is not available, we approximated $\Sigma^{\prime \prime}$ at high energies by the scaled, calculated partial DOS (PDOS) to match the the experimental data, noting that $\Sigma^{\prime \prime}$ is proportional to PDOS as in equation (3). The resulting $\Sigma^{\prime \prime}$ for the NB along the $K$ $M$ direction is plotted in panel (c). $\Sigma^{\prime}$ is then obtained by a Hilbert transformation of the symmetrized $\Sigma^{\prime \prime}$ and plotted in panel (d). The $\lambda_{N B}$ value obtained from $\Sigma^{\prime}$ is about 0.12 , which is much smaller than the previously estimated value from ARPES 8 . For the $\mathrm{K}-\Gamma$ direction, $\lambda$ was 0.2 . Therefore, we set 0.2 as the upper bound for EPC constant $\lambda$. This value is very small compared to $\lambda$ values in typical metals as expected for semi-metals with very small DOS at the Fermi energy.

Authors acknowledge helpful discussions with J. H. Han. This work is supported by the KICOS through a grant provided by MOST in M60602000008-06E020000800, by KOSEF through CSCMR, and also by MOST through GPP. ALS is operated by the DOE's Office of BES.
* Electronic address: cykim@phya.yonsei.ac.kr

1 A. F. Hebard et al., Nature 350, 600 (1991).

2 Z. K. Tang et al., Science 292, 2462 (2001).

3 M. Kociak et al., Phys. Rev. Lett. 86, 2416 (2001).

4 T. E. Weller et al., Nature Physics 1, 39 (2005).

5 N. Emery et al., Phys. Rev. Lett. 95, 087003 (2005).

${ }^{6}$ K. S. Novoselov et al., Proc. Natl. Acad. Sci. U.S.A. 102, 10451 (2005).

7 S. Y. Zhou et al., Nature Physics 2, 595 (2006).

${ }^{8}$ K. Sugawara et al., Phys. Rev. Lett. 98, 036801 (2007).
9 A. R. Law, J. J. Barry, and H. P. Hughes, Phys. Rev. B 28, 5332 (1983).

10 T. Takahashi, H. Tokailin, and T. Sagawa, Phys. Rev. B 32, 8317 (1985).

11 A. R. Law, M. T. Johnson, and H. P. Hughes, Phys. Rev. B 34, 4289 (1986).

12 F. Maeda et al., Phys. Rev. B 37, 4482 (1988).

13 I. R. Collins, P. T. Andrews, and A. R. Law, Phys. Rev. B 38, 13348 (1988).

14 V. N. Strocov, A. Charrier, and J. -M. Themlin, Phys. Rev. 
B 64, 075105 (2001).

15 T. Kihlgren, T. Balasubramanian, L. Walldén, and R. Yakimova, Phys. Rev. B 66, 235422 (2002).

16 A. Bostwick et al., Nature Phys. 3, 36 (2007).

17 T. Ohta et al., Science 313, 951 (2006).

18 C. Fretigny, D. Marchand, and M. Laguës, Phys. Rev. B 32, 8462 (1985).

19 S. L. Molodtsov et al., Phys. Rev. B 53, 16621 (1996).

20 S. Xu et al., Phys. Rev. Lett. 76, 483 (1996).

${ }^{21}$ G. Moos et al., Phys. Rev. Lett. 87, 267402 (2001).

22 D. Sánchez-Portal et al., Int. J. Quantum Chem. 65, 453 (1997).

23 B. Feuerbacher and B. Fitton, Phys. Rev. Lett. 26, 840 (1971).

${ }^{24}$ K. Sugawara, T. Sato, S. Souma, T. Takahashi, and H. Suematsu, Phys. Rev. B 73, 045124 (2006)

25 S. Tanuma, C. J. Powell, and D. R. Penn, Surface and
Interface Analysis 17, 911 (1991).

26 G. Grimvall, The Electron-Phonon Interaction in Metals, Selected Topics in Solid State Physics, edited by E. Wohlfarth, North-Holland, New York, 1981.

27 M. Hengsberger et al., Phys. Rev. Lett. 83, 592 (1999).

28 T. Valla et al., Phys. Rev. Lett. bf 83, 2085 (1999).

29 P. Lautenschlager, P. B. Allen and M. Cardona, Phys. Rev. B 33, 5501 (1986)

30 See supplementary information at EPAPS.

31 To be precise, the Lorentzian width of the Voigt function should be varied as a function of energy in convolving the model function. That is, one has to do energy dependent convolution for each EDC.

32 C. D. Spataru et al., Phys. Rev. Lett. 87, 246405 (2001).

33 E. Rotenberg et al., unpublished. 
This file contains supplementary information on the the self energies due to the electron-phonon coupling (EPC) and electron hole pair creation in graphene and graphite which have characteristic linear electronic density of states near the Fermi energy (linear DOS). We first investigate the scattering process through phonons by using schematic diagrams for the single (graphene) and double (graphite) conical band structures. It allows one to intuitively understand how characteristic linear DOS contributes to the scattering rate of photo-hole. Then we discuss the scattering process by electron-hole pair creation by considering the available phase space for the process. We show that this process gives little contribution to the scattering rate of a photo-hole.

\section{SCATTERING RATE DUE TO ELECTRON-PHONON COUPLING}

\section{A. Single band case}

In this case, we assume a conical band structure with the Fermi energy at the apex of the cone. There also is an unoccupied conical band above the Fermi energy, forming a Dirac-cone-like band structure as shown in Fig. 1(a). Assume an Einstein phonon at energy $\omega_{0}$. If a photo-hole with momentum $\mathbf{k}$ (filled circle) is created by a photon as shown in Fig. 1(a), it can be filled by an electron with energy of $\omega_{k^{\prime}}=\omega_{k}-\omega_{0}$ and momentum $\mathbf{k}^{\prime}=\mathbf{k}-\mathbf{q}$ (empty circles) where $\mathbf{q}$ is the phonon momentum. We further assumed for simplicity the scattering amplitude $g$ is momentum independent. In that case, the scattering rate is proportional to the number of such $\mathbf{k}^{\prime}$ states, thus the DOS at $\omega_{k}-\omega_{0}$. Note that if the binding energy of the photo-hole is smaller than the phonon energy $\omega_{0}$, the scattering cannot occur because there are no electrons with sufficient energy to emit a phonon. Therefore, the self energy of photo-hole as a function of the binding energy is proportional to $\mathcal{D}\left(\omega_{k}-\omega_{0}\right)$ and looks like the schematic shown in Fig. 1(b).

\section{B. Double band case}

For the double band case, another conical band exists $\omega_{B}$ below the Fermi energy as shown in Fig. 2(a). One needs to investigate the cases where the initial photohole is in non-bonding and bonding bands separately. We again count the the number of available electrons that can fill the photo-hole. We first consider the case in which the photo-hole is created in non-bonding band. If the binding energy of the photo-hole is smaller than $\omega_{B}+\omega_{0}$, the decay rate (the number of electrons that can fill the photo-hole) will be the same as that in the single band case. When the binding energy becomes larger than $\omega_{B}+\omega_{0}$, the additional electrons (triangles in Fig. 2(a)) in the second band begin to contribute to the scattering process. This additional contribution is proportional
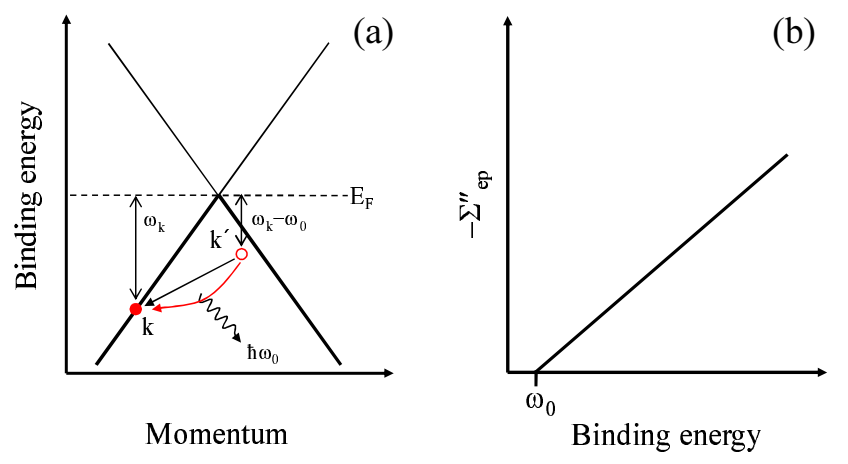

FIG. 1: (a) Schematic diagram of the photo-hole decay by emitting a phonon in the single band case. $\omega_{0}$ is phonon energy and $\mathbf{k}$ and $\mathbf{k}^{\prime}$ are initial and final photo-hole states, respectively. (b) Schematic of the resulting decay rate (the imaginary part of self energy) vs binding energy. It is zero up to $\omega_{0}$ and then increases linearly.
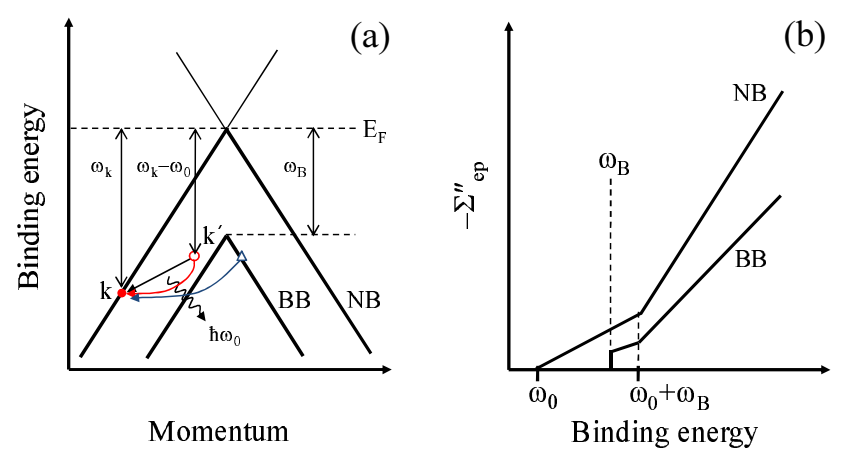

FIG. 2: (a) Schematic diagram of the photo-hole decay in the double band case. $\omega_{B}$ is the energy of the apex of the lower conical band. (b) The imaginary parts of the self energies vs binding energy for photo-holes created in non-bonding and bonding bands.

to the partial DOS of the second band at $\omega_{k}-\omega_{B}$ and thus linearly increases. Note that the additional slope of the linear increase depends on the inter-band scattering amplitude $g_{i j}(i \neq j)$. The resulting decay rate is schematically shown in Fig. 2(b). We now investigate the case for a photo-hole in the bonding band. If the energy of the photo-hole is larger than $\omega_{B}$ but smaller than $\omega_{B}+\omega_{0}$, the photo-hole can be filled with only electrons in non-bonding band via inter-band transition. Meanwhile the photo-hole can decay via inter- and intra-band transitions when the energy is larger than $\omega_{B}+\omega_{0}$. The resulting decay rate is also schematically shown in Fig. 2(b). If the electron-phonon couplings are different for bonding and non-bonding bands, the overall slopes of self energies will be different as shown in Fig 2.(b). 


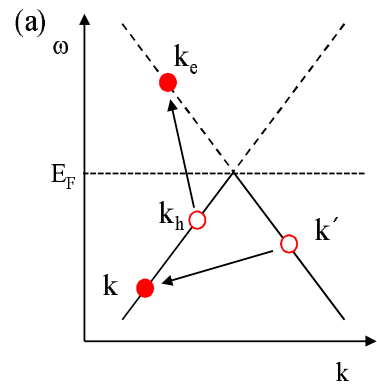

(b)

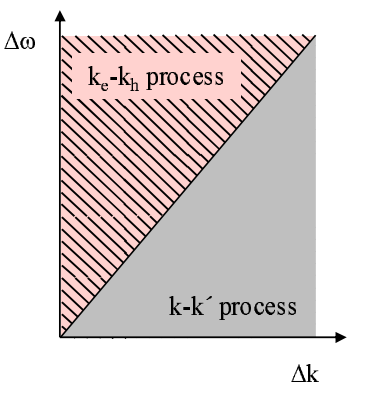

FIG. 3: (a) Schematic of electron-hole creation $\left(\mathbf{k}_{e}-\mathbf{k}_{h}\right)$ and transition of a photo-hole $\left(\mathbf{k}_{e}-\mathbf{k}^{\prime}\right)$ in a conical band structure. (b) Available phase spaces for $\mathbf{k}_{e}-\mathbf{k}_{h}$ (hatched region) as well as $\mathbf{k}_{e}-\mathbf{k}^{\prime}$ (grey region) processes. Two regions overlap only at the boundary.

\section{AVAILABLE PHASE SPACE FOR ELECTRON-HOLE PAIR CREATION}

A photo-hole with the momentum $\mathbf{k}$ is created in the Dirac cone as shown in Fig 3.(a). It may go through a transition to a $\mathbf{k}^{\prime}$ state by creating an electron-hole pair (denoted as $\mathbf{k}_{\mathbf{e}}$ and $\mathbf{k}_{\mathbf{h}}$, respectively in Fig 3.(a)). If we plot the energy difference $\left(\Delta \omega=\omega_{k}-\omega_{k^{\prime}}\right)$ as a function of momentum difference $\Delta \mathbf{k}=\mathbf{k}-\mathbf{k}^{\prime}$, the possible transitions occupy the lower right of the $\Delta \omega-\Delta \mathbf{k}$ phase space as shown in Fig. 3(b) (grey). In a similar way, we can go through the same process for the electronhole pair creation. One can show that $\Delta \omega=\omega_{e}-\omega_{h}$ and $\Delta \mathbf{k}=\mathbf{k}_{\mathbf{e}}-\mathbf{k}_{\mathbf{h}}$ of the electron-hole creation processes occupy the upper left of the phase space (hatched). We note that only the boundary is shared by two regions. Energy and momentum conservations enforce the decay through the electron-hole pair creation to occur in the phase space points that belong to both regions. Therefore, the available phase space volume for the decay is zero and thus the transition rate. 